Article

\title{
Synthesis and Thermal Characteristic of Liquid Crystalline Polyoxetane Containing Trans-Stilbene Side Group
}

\author{
Li-Chuan $\mathrm{Wu}^{1}{ }^{1}$, Cheng-Chih Chen ${ }^{2}$ and Chih-Hung Lin ${ }^{2,3, *(D)}$ \\ 1 Department of Applied Chemistry and Material Sciences, Fooyin University, 151 Jinxue Road, Daliao, \\ Kaohsiung City 83102, Taiwan; SC023@fy.edu.tw \\ 2 Center for General Education, Chang Gung University of Science and Technology, 261 Wen-Hwa 1st Road, \\ Kwei-Shan, Tao-Yuan 33303, Taiwan; ccchen@gw.cgust.edu.tw \\ 3 Research Center for Food and Cosmetic Safety and Research Center for Chinese Herbal Medicine, \\ Chang Gung University of Science and Technology, Kweishan, Tao-Yuan 33303, Taiwan \\ * Correspondence: chlin@mail.cgust.edu.tw or chihhung5622@gmail.com
}

Received: 21 December 2019; Accepted: 6 January 2020; Published: 10 January 2020

check for updates

\begin{abstract}
A series of fourteen liquid crystalline monomers and polyoxetanes containing trans-biphenyl side group have been successfully synthesized. The thermal and mesomorphic properties of monomers (1M 14M) and polymers (1P 14P) are measured using DSC, POM, and X-ray. All of the series monomers present enantiotropic smectic $H$ and smectic $G$ phase and the series polymers show enantiotropic smectic A phase which three polymers contained exhibit smectic E. Polyoxetanes have been used as a cationic ring-opening polymerization of oxetane monomers bearing a pendant trans-stilbene mesogenic unit including different spacer length and terminal alkyl length.
\end{abstract}

Keywords: polyoxetane; liquid crystal; stilbene

\section{Introduction}

The first thermotropic side-chain liquid crystalline polymers were synthesized by Finkelmann and Rehage [1,2]. They had already understood the main factors in the formation of the liquid crystal phase of compounds. The liquid crystal properties of the compound were affected by the backbone liquid crystal structure, the mesogenic unit, the tail group, and the spacer length. The side-chain liquid crystal polymer integrated the properties of the liquid crystal and the polymer properties. They had potential applications in optical data storage, piezoelectric transducer, nonlinear optics, and gas or liquid chromatography stationary phases [3-7].

In the past few decades, a large number of side-chain liquid crystal polymers had been synthesized [8-10]. They combined the many different backbone types (such as methacrylates, acrylates, siloxanes, epoxides, ethylenes, etc.) and the vast number of mesogenic units available. Kawakami et al. reported the first example of a cationic ring-opening polymerized side-chain liquid crystalline polyoxetane [11-16].

According to the experimental results, polyoxetane flexibility had more than polyacrylate and polymethacrylate. The polymerization of the side-chain liquid crystalline polyoxetane molecular weight distribution (MWD) is less than 1.3.

The purpose of this research is to show the synthesis of a new series of side-chain liquid crystalline polyoxetanes containing the trans-stilbene mesogenic side group. The effects of terminal alkyl length and spacer length on the properties of mesophases exhibited are discussed. 


\section{Materials and Methods}

\subsection{Instruments}

${ }^{1} \mathrm{H}-\mathrm{NMR}(400 \mathrm{MHz})$ spectra were measured using a Bruker AM 400 instrument (Bruker, Daltonik, Germany). The thermal transitions and the anisotropic textures were measured using a Carl-Ziess Axiphot polarized optical microscope (Carl-Ziess, Jena, Germany) and a Mettler FP82 hot stage (Mettler, Switzerland). Differential scanning calorimeter (DSC) was recorded on a Seiko SSC/5200 (Seiko, New Castle, DE, USA) with determined compounds of thermal transitions and thermodynamic parameters equipped with a cooling accessory. Thermal stability was measured using a Seiko TG/DTA 200 thermal gravimetric analyzer (Seiko, New Castle, DE, USA). X-ray diffraction by liquid crystals was measured using a Riraku powder diffractometer (Riraku, Austin, TX, USA).

\subsection{Synthesis}

The intermediates and targets compound synthetic routes were represented in Scheme 1. TLC and ${ }^{1} \mathrm{H}-\mathrm{NMR}$ spectroscopy were verified as the chemical structures and purity of the intermediates and target compounds. The synthesis methods and analysis of each product are described below.

3-[(3-Bromopropoxy)methyl]-3-methyloxetane (1a)

3-[(4-Bromobutoxy)methyl]-3-methyloxetane (1b)

3-[(5-Bromopentoxy)methyl]-3-methyloxetane (1c)

3-[(6-Bromohexoxy)methyl]-3-methyloxetane (1d)

3-[(12-Bromododecoxy)methyl]-3-methyloxetane (1e)

All five compounds were prepared by the same method. Taking compound 1d as an example, the synthesis is described below.

3-(Hydroxymethyl)-3-methyloxetane (10.0 g, $0.098 \mathrm{~mol})$, dibromohexane (73.2 g, $0.299 \mathrm{~mol})$, and hexane $(120 \mathrm{~mL})$ was added to a stirred solution of sodium hydroxide $(64.7 \mathrm{~g}, 1.618 \mathrm{~mol})$ in 150 $\mathrm{mL}$ of water. Then, tetrabutylammonium bromide $(1.0 \mathrm{~g})$ was added to the solution. The solution was stirred for $12 \mathrm{~h}$ at room temperature, then heated to reflux for $0.5 \mathrm{~h}$. The reaction solution cooled to room temperature, $1000 \mathrm{~mL}$ of water was added, and the organic layer was extracted three times with hexane. The extraction solution was dried through anhydrous magnesium sulfate and after removal of the solvent under reduced pressure. The crude product was purified by distillation, to yield $20.85 \mathrm{~g}$ $(80.3 \%)$ of a colorless transparent liquid. ${ }^{1} \mathrm{H}-\mathrm{NMR}\left(300 \mathrm{MHz}, \mathrm{CDCl}_{3}, \delta, \mathrm{ppm}\right): 1.30\left(\mathrm{~s}, 3 \mathrm{H},-\mathrm{CH}_{3}\right.$ on the oxetane ring), $1.46-1.90\left(\mathrm{~m}, 8 \mathrm{H},-\mathrm{OCH}_{2}\left(\mathrm{CH}_{2}\right)_{4} \mathrm{CH}_{2}-\right)$, 3.39-3.48 (m, 6H, $\left.-\mathrm{CH}_{2} \mathrm{OCH}_{2}\left(\mathrm{CH}_{2}\right)_{4} \mathrm{CH}_{2} \mathrm{Br}\right)$, $4.34,4.51$ ( $\mathrm{AB}$ quartet, each $2 \mathrm{H},-\mathrm{CH}_{2}-\mathrm{O}$ on the oxetane ring).

3-[[3-(4-Hydroxybenzaldehyde)propoxy]methyl]-3-methyl oxetane (2a)

3-[[4-(4-Hydroxybenzaldehyde)butoxy]methyl]-3-methyl oxetane (2b)

3-[[5-(4-Hydroxybenzaldehyde)pentoxy]methyl]-3-methyl oxetane (2c)

3-[[6-(4-Hydroxybenzaldehyde)hexoxy]methyl]-3-methyl oxetane (2d)

3-[[12-(4-Hydroxybenzaldehyde)dodecanoxy]methyl]-3-methyl oxetane (2e)

4-Butoxy-benzaldehyde (3a)

4-Pentoxy-benzaldehyde (3b)

4-Hexoxy-benzaldehyde (3c)

4-Heptoxy-benzaldehyde (3d)

4-Octoxy-benzaldehyde (3e)

All ten compounds were prepared by the same method. Taking compound $\mathbf{2 d}$ as an example, the synthesis is described below. 
<smiles>CC1(CO)COC1</smiles>

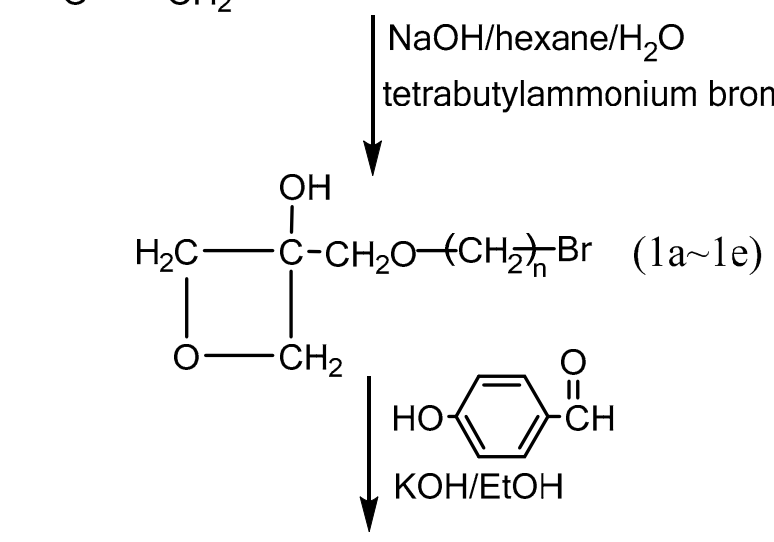

$\mathrm{NaOH} /$ hexane $/ \mathrm{H}_{2} \mathrm{O}$ bromide<smiles>O=C[14C](=O)[14C](=O)c1ccc(O)cc1</smiles>

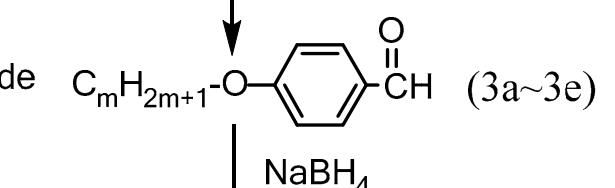<smiles>CC1(COCCOc2ccc(C=O)cc2)COC1</smiles>
$\mathrm{P}\left(\mathrm{OC}_{2} \mathrm{H}_{5}\right)_{3}$<smiles>CCOCc1ccc(OCC)c(OCC)c1</smiles>

$(6 a \sim 6 e)$<smiles>CCOc1ccc(/C=C/c2ccc(OCC)cc2)cc1</smiles>

15-crown-5 ether $\mathrm{NaH} / \mathrm{THF}$<smiles>CC(C)(C)COC(C)(C)C</smiles>

$(1 \mathrm{M} \sim 14 \mathrm{M})$

\begin{tabular}{|c|c|c|c|c|c|c|c|c|c|c|c|c|c|c|}
\hline monomer & $1 \mathrm{M}$ & $2 \mathrm{M}$ & $3 \mathrm{M}$ & $4 \mathrm{M}$ & $5 \mathrm{M}$ & $6 \mathrm{M}$ & $7 \mathrm{M}$ & $8 \mathrm{M}$ & $9 \mathrm{M}$ & $10 \mathrm{M}$ & $11 \mathrm{M}$ & $12 \mathrm{M}$ & $13 \mathrm{M}$ & $14 \mathrm{M}$ \\
\hline polymer & $1 \mathrm{P}$ & $2 \mathrm{P}$ & $3 \mathrm{P}$ & $4 \mathrm{P}$ & $5 \mathrm{P}$ & $6 \mathrm{P}$ & $7 \mathrm{P}$ & $8 \mathrm{P}$ & $9 \mathrm{P}$ & $10 \mathrm{P}$ & $11 \mathrm{P}$ & $12 \mathrm{P}$ & $13 \mathrm{P}$ & $14 \mathrm{P}$ \\
\hline $\mathrm{n}$ & 3 & 4 & 5 & 6 & 6 & 6 & 6 & 6 & 6 & 12 & 12 & 12 & 12 & 12 \\
\hline $\mathrm{m}$ & 1 & 1 & 1 & 1 & 4 & 5 & 6 & 7 & 8 & 4 & 5 & 6 & 7 & 8 \\
\hline
\end{tabular}

Scheme 1. Synthesis of monomers $\mathbf{1 M} \sim \mathbf{1 4 M}$ and polymers $\mathbf{1 P} \sim \mathbf{1 4 P}$.

4-Hydroxybenzaldehyde ( $4.45 \mathrm{~g}, 0.036 \mathrm{~mol})$ was added to a stirred solution of potassium hydroxide $(2.04 \mathrm{~g}, 0.036 \mathrm{~mol})$ and potassium iodide $(0.2 \mathrm{~g})$ in $100 \mathrm{~mL}$ of $95 \%$ ethanol. Compound $\mathbf{1 d}(8.00 \mathrm{~g}$, $0.030 \mathrm{~mol}$ ) was added dropwise after the solution mentioned above was refluxed for $1 \mathrm{~h}$. The solution refluxed for $12 \mathrm{~h}$ and then cooled to room temperature. The solution was extracted with water and ethyl acetate. The extraction solution was washed with $10 \% \mathrm{KOH}$ solution three times and dried through anhydrous magnesium sulfate. After removal of the solvent under reduced pressure, the crude product 
was purified by column chromatography on silica gel using ethyl acetate/hexane as eluent to yield 2.42 $\mathrm{g}(82.5 \%)$ of light yellow liquid. ${ }^{1} \mathrm{H}-\mathrm{NMR}\left(300 \mathrm{MHz}, \mathrm{CDCl}_{3}, \delta, \mathrm{ppm}\right): 1.30\left(\mathrm{~s}, 3 \mathrm{H},-\mathrm{CH}_{3}\right.$ on the oxetane ring), $1.40-1.82\left(\mathrm{~m}, 8 \mathrm{H},-\mathrm{OCH}_{2}\left(\mathrm{CH}_{2}\right)_{4} \mathrm{CH}_{2}-\right), 3.46\left(\mathrm{~m}, 4 \mathrm{H},-\mathrm{CH}_{2} \mathrm{O}-\right), 4.04\left(\mathrm{t}, 2 \mathrm{H},-\mathrm{CH}_{2}-\mathrm{OPh}\right), 4.33$, 4.49 (AB quartet, each $2 \mathrm{H},-\mathrm{CH}_{2}-\mathrm{O}$ on the oxetane ring), $6.96(\mathrm{~d}, 2 \mathrm{H}$, aromatic protons), $7.81(\mathrm{~d}, 2 \mathrm{H}$, aromatic protons), 9.87 (s, $1 \mathrm{H}$, aldehyde protons).

4-Butoxy-benzyl alcohol (4a)

4-Pentoxy-benzyl alcohol (4b)

4-Hexoxy-benzyl alcohol (4c)

4-Heptoxy-benzyl alcohol (4d)

4-Octoxy-benzyl alcohol (4e)

Taking compound $4 \mathrm{c}$ as an example, the synthesis is described below.

A solution of sodium tetrahydridoborate $(3.28 \mathrm{~g}, 0.087 \mathrm{~mol})$ in $2 \mathrm{~mL}$ of $0.45 \mathrm{~N}$ sodium hydroxide with $28 \mathrm{~mL}$ water was slowly added dropwise to 4-Hexoxy-benzaldehyde (14.9 $\mathrm{g}, 0.072 \mathrm{~mol})$ with methanol $(150 \mathrm{~mL})$ solution. The solution was stirred $3 \mathrm{~h}$ at room temperature. Remove most of the methanol by distillation. The solution was extracted with ether and aqueous of dilute acid solution $(50 \mathrm{~mL})$. The organic phase was washed with $2 \%$ aqueous of sodium bicarbonate, saturated aqueous of sodium chloride, and dried through anhydrous magnesium sulfate. The crude product was purified by column chromatography on silica gel using $n$-hexane/ethyl acetate as eluent to yield $13.8 \mathrm{~g}(92.1 \%)$ of colorless liquid. ${ }^{1} \mathrm{H}-\mathrm{NMR}\left(300 \mathrm{MHz}, \mathrm{CDCl}_{3}, \delta, \mathrm{ppm}\right): 0.89\left(\mathrm{t}, 3 \mathrm{H},-\mathrm{CH}_{3}\right), 1.31-1.80$ $\left(\mathrm{m}, 8 \mathrm{H},-\mathrm{OCH}_{2}\left(\mathrm{CH}_{2}\right)_{4} \mathrm{CH}_{3}\right), 3.96\left(\mathrm{t}, 2 \mathrm{H},-\mathrm{OCH}_{2}\left(\mathrm{CH}_{2}\right)_{4}-\right), 4.66\left(\mathrm{~s}, 2 \mathrm{H}, \mathrm{Ph}-\mathrm{CH}_{2} \mathrm{Cl}\right), 6.89(\mathrm{~d}, 2 \mathrm{H}$, aromatic protons), 7.30 (d, 2H, aromatic protons).

4-Butoxy-benzyl chloride (5a)

4-Pentoxy-benzyl chloride (5b)

4-Hexoxy-benzyl chloride $(5 \mathrm{c})$

4-Heptoxy-benzyl chloride (5d)

4-Octoxy-benzyl chloride (5e)

All five compounds were prepared by the same method. Taking compound $5 c$ as an example, the synthesis is described below.

4-Hexoxy-benzyl alcohol (5 g, $0.024 \mathrm{~mol})$ was reacted with excess thionyl chloride (15 mL, $0.0206 \mathrm{~mol}$ ) in $50 \mathrm{~mL}$ of methylene chloride. The solution was stirred at ice bath for $6 \mathrm{~h}$. Then, added with water $(30 \mathrm{~mL})$ into the solution. The organic phase was washed with $10 \%$ aqueous of sodium bicarbonate, saturated aqueous of sodium chloride, and dried through anhydrous magnesium sulfate. The crude product was purified by column chromatography on silica gel using n-hexane/ethyl acetate as eluent to yield $4.58 \mathrm{~g}(84.3 \%)$ of light-yellow liquid. ${ }^{1} \mathrm{H}-\mathrm{NMR}\left(300 \mathrm{MHz}, \mathrm{CDCl}_{3}, \delta, \mathrm{ppm}\right): 0.89(\mathrm{t}$, $\left.3 \mathrm{H},-\mathrm{CH}_{3}\right), 1.31-1.80\left(\mathrm{~m}, 8 \mathrm{H},-\mathrm{OCH}_{2}\left(\mathrm{CH}_{2}\right)_{4} \mathrm{CH}_{3}\right), 3.96\left(\mathrm{t}, 2 \mathrm{H},-\mathrm{OCH}_{2}\left(\mathrm{CH}_{2}\right)_{4}-\right), 4.66\left(\mathrm{~s}, 2 \mathrm{H}, \mathrm{Ph}-\mathrm{CH}_{2} \mathrm{Cl}\right)$, $6.89(\mathrm{~d}, 2 \mathrm{H}$, aromatic protons), 7.30 (d, 2H, aromatic protons).

Diethyl[(4-butoxy)benzyl]phosphonate (6a)

Diethyl[(4-pentoxy)benzyl]phosphonate (6b)

Diethyl[(4-hexoxy)benzyl]phosphonate (6c)

Diethyl[(4-heptoxy)benzyl]phosphonate (6d)

Diethyl[(4-octoxy)benzyl]phosphonate (6e)

All five compounds were prepared by the same method. Taking compound $6 \mathrm{c}$ as an example, the synthesis is described below.

4-Hexoxy-benzyl chloride $(5.00 \mathrm{~g}, 0.022 \mathrm{~mol})$ was injected by triethyl phosphite $(11.0 \mathrm{~g}, 0.066 \mathrm{~mol})$ with a syringe under nitrogen. The reaction mixture was refluxed for $12 \mathrm{~h}$, then, was purified by distillation under reduced pressure to yield $5.89 \mathrm{~g}(81.6 \%)$ of colorless liquid. ${ }^{1} \mathrm{H}-\mathrm{NMR}(300 \mathrm{MHz}$, $\left.\mathrm{CDCl}_{3}, \delta, \mathrm{ppm}\right): 0.89\left(\mathrm{t}, 3 \mathrm{H},-\mathrm{CH}_{3}\right), 1.22\left(\mathrm{~m}, 6 \mathrm{H}, \mathrm{P}\left(\mathrm{OCH}_{2} \mathrm{CH}_{3}\right)_{2}, 1.31-1.79\left(\mathrm{~m}, 8 \mathrm{H},-\mathrm{OCH}_{2}\left(\mathrm{CH}_{2}\right)_{4} \mathrm{CH}_{3}\right)\right.$, $3.05\left(\mathrm{~d}, 2 \mathrm{H}, \mathrm{PCH}_{2} \mathrm{Ph}\right), 3.93-4.01\left(\mathrm{~m}, 6 \mathrm{H},-\mathrm{OCH}_{2}\left(\mathrm{CH}_{2}\right)_{4}-\right.$ and $\left.\mathrm{P}\left(\mathrm{OCH}_{2} \mathrm{CH}_{3}\right)_{2}\right), 6.89(\mathrm{~d}, 2 \mathrm{H}$, aromatic protons), $7.30(\mathrm{~d}, 2 \mathrm{H}$, aromatic protons).

3-[3-(Trans-4'-methoxystilben-4-yloxy)propoxymethyl]-3-methyl oxetane (1M) 
3-[4-(Trans-4'-methoxystilben-4-yloxy)butoxymethyl]-3-methyl oxetane (2M)

3-[5-(Trans-4'-methoxystilben-4-yloxy)pentoxymethyl]-3-methyl oxetane (3M)

3-[6-(Trans-4'-methoxystilben-4-yloxy)hexoxymethyl]-3-methyl oxetane (4M)

3-[6-(Trans-4'-butoxystilben-4-yloxy)hexoxymethyl]-3-methyl oxetane (5M)

3-[6-(Trans-4'-pentoxystilben-4-yloxy)hexoxymethyl]-3-methyl oxetane (6M)

3-[6-(Trans-4'-hexoxystilben-4-yloxy)hexoxymethyl]-3-methyl oxetane (7M)

3-[6-(Trans-4'-heptoxystilben-4-yloxy)hexoxymethyl]-3-methyl oxetane (8M)

3-[6-(Trans-4'-octoxystilben-4-yloxy)hexoxymethyl]-3-methyl oxetane (9M)

3-[12-(Trans-4' -butoxystilben-4-yloxy)dodecoxymethyl]-3-methyl oxetane (10M)

3-[12-(Trans-4' -pentoxystilben-4-yloxy)dodecoxymethyl]-3-methyl oxetane (11M)

3-[12-(Trans-4'-hexoxystilben-4-yloxy)dodecoxymethyl]-3-methyl oxetane (12M)

3-[12-(Trans-4'-heptoxystilben-4-yloxy)dodecoxymethyl]-3-methyl oxetane (13M)

3-[12-(Trans-4'-octoxystilben-4-yloxy)dodecoxymethyl]-3-methyl oxetane (14M)

All fourteen monomers $\mathbf{1} \mathbf{M} \sim \mathbf{1 4} \mathbf{M}$ were prepared by the same method. Taking monomer $\mathbf{7} \mathbf{M}$ as an example, the synthesis is described below.

Sodium hydride $(0.33 \mathrm{~g}, 0.013 \mathrm{~mol})$ dissolved in dry THF $(50 \mathrm{~mL})$ on the brown flask, 13-crown-5-ether $(30 \mathrm{mg}$ ) was added to react under nitrogen in the ice bath. Then, a solution of compound $2 \mathrm{~d}(2.52 \mathrm{~g}, 0.008 \mathrm{~mol})$ and compound $6 \mathrm{~d}(2.6 \mathrm{~g}, 0.008 \mathrm{~mol})$ was added dropwise to a stirred mixture. The reaction mixture was stirred for $12 \mathrm{~h}$ at room temperature. The reaction solution mixture was poured into ice water. The solution was filtered, the remaining yellow solid was recrystallized from dimethyl formamide to yield $1.62 \mathrm{~g}$ of light-yellow solid.

The ${ }^{1} \mathrm{H}-\mathrm{NMR}$ spectrometer, the product yield, and element analysis of monomers $\mathbf{1} \mathbf{M} \sim \mathbf{1 4} \mathbf{M}$ were as follows.

Compound 1M: Yield: $40.7 \%$; ${ }^{1} \mathrm{H}-\mathrm{NMR}\left(300 \mathrm{MHz}, \mathrm{CDCl}_{3}, \delta, \mathrm{ppm}\right): 1.28\left(\mathrm{~s}, 3 \mathrm{H},-\mathrm{CH}_{3}\right.$ on the oxetane ring), $2.03\left(\mathrm{t}, 2 \mathrm{H},-\mathrm{OCH}_{2} \mathrm{CH}_{2}-\right), 3.48\left(\mathrm{~s}, 2 \mathrm{H},-\mathrm{CH}_{2} \mathrm{O}\left(\mathrm{CH}_{2}\right)_{3}-\right), 3.62\left(\mathrm{t}, 2 \mathrm{H},-\mathrm{CH}_{2} \mathrm{OCH}_{2}-\right), 3.80(\mathrm{~s}$, $\left.3 \mathrm{H},-\mathrm{PhOCH}_{3}\right), 4.04\left(\mathrm{t}, 2 \mathrm{H},-\mathrm{CH}_{2} \mathrm{CH}_{2} \mathrm{OPh}\right), 4.32,4.48$ ( $\mathrm{AB}$ quartet, each $2 \mathrm{H},-\mathrm{CH}_{2}-$ on the oxetane ring), 6.84, $7.38\left(\mathrm{~m}, 6 \mathrm{H}, 4 \mathrm{H}\right.$, stilbene protons); element analysis: Calc. for $\mathrm{C}_{23} \mathrm{H}_{28} \mathrm{O}_{4}$ : C 75.00, H 7.61, O 17.39; found C 75.00, H 7.75, O $17.25 \%$.

Compound 2M: Yield: $38.6 \%$; ${ }^{1} \mathrm{H}-\mathrm{NMR}\left(300 \mathrm{MHz}, \mathrm{CDCl}_{3}, \delta, \mathrm{ppm}\right): 1.31\left(\mathrm{~s}, 3 \mathrm{H},-\mathrm{CH}_{3}\right.$ on the oxetane ring), $1.79\left(\mathrm{~m}, 4 \mathrm{H},-\mathrm{OCH}_{2}\left(\mathrm{CH}_{2}\right)_{2}-\right), 3.49\left(\mathrm{t}, 4 \mathrm{H},-\mathrm{CH}_{2} \mathrm{OCH}_{2}-\right), 3.82\left(\mathrm{~s}, 3 \mathrm{H},-\mathrm{PhOCH}_{3}\right), 3.98(\mathrm{t}, 2 \mathrm{H}$, $-\mathrm{CH}_{2} \mathrm{CH}_{2} \mathrm{OPh}$ ), 4.34, 4.50 (AB quartet, each $2 \mathrm{H},-\mathrm{CH}_{2}$ - on the oxetane ring), 6.85, $7.39(\mathrm{~m}, 6 \mathrm{H}, 4 \mathrm{H}$, stilbene protons); element analysis: Calc. for $\mathrm{C}_{24} \mathrm{H}_{30} \mathrm{O}_{4}$ : C 75.39, $\mathrm{H}$ 7.85, O 16.75; found C 75.46, H 7.99, O 16.45\%.

Compound 3M: Yield: $33.4 \%,{ }^{1} \mathrm{H}-\mathrm{NMR}\left(300 \mathrm{MHz}, \mathrm{CDCl}_{3}, \delta, \mathrm{ppm}\right): 1.34\left(\mathrm{~s}, 3 \mathrm{H},-\mathrm{CH}_{3}\right.$ on the oxetane ring), $1.58-1.87\left(\mathrm{~m}, 6 \mathrm{H},-\mathrm{OCH}_{2}\left(\mathrm{CH}_{2}\right)_{3}-\right), 3.51\left(\mathrm{t}, 4 \mathrm{H},-\mathrm{CH}_{2} \mathrm{OCH}_{2}-\right), 3.85\left(\mathrm{~s}, 3 \mathrm{H},-\mathrm{PhOCH}_{3}\right)$, $3.99\left(\mathrm{t}, 2 \mathrm{H},-\mathrm{CH}_{2} \mathrm{CH}_{2} \mathrm{OPh}\right), 4.38,4.53$ (AB quartet, each $2 \mathrm{H},-\mathrm{CH}_{2}-$ on the oxetane ring), 6.85, 7.43 (m, $6 \mathrm{H}, 4 \mathrm{H}$, stilbene protons); element analysis: Calc. for $\mathrm{C}_{25} \mathrm{H}_{32} \mathrm{O}_{4}: \mathrm{C} 75.76, \mathrm{H} 8.08, \mathrm{O} 16.16$; found $\mathrm{C}$ 75.52, H 8.15, O $16.33 \%$.

Compound 4M: Yield: $30.8 \% ;{ }^{1} \mathrm{H}-\mathrm{NMR}\left(300 \mathrm{MHz}, \mathrm{CDCl}_{3}, \delta, \mathrm{ppm}\right): 1.31\left(\mathrm{~s}, 3 \mathrm{H},-\mathrm{CH}_{3}\right.$ on the oxetane ring), $1.47-1.85\left(\mathrm{~m}, 8 \mathrm{H},-\mathrm{OCH}_{2}\left(\mathrm{CH}_{2}\right)_{4}-\right), 3.46\left(\mathrm{t}, 4 \mathrm{H},-\mathrm{CH}_{2} \mathrm{OCH}_{2}-\right), 3.81\left(\mathrm{~s}, 3 \mathrm{H},-\mathrm{PhOCH}_{3}\right), 3.99$ $\left(\mathrm{t}, 2 \mathrm{H},-\mathrm{CH}_{2} \mathrm{CH}_{2} \mathrm{OPh}\right), 4.34,4.50$ ( $\mathrm{AB}$ quartet, each $2 \mathrm{H},-\mathrm{CH}_{2}$ - on the oxetane ring), $6.87,7.26(\mathrm{~m}, 6 \mathrm{H}$, $4 \mathrm{H}$, stilbene protons); element analysis: Calc. for $\mathrm{C}_{26} \mathrm{H}_{34} \mathrm{O}_{4}$ : C 76.10, $\mathrm{H} 8.29, \mathrm{O} 15.61$; found C 75.82, $\mathrm{H}$ 8.47, O $15.71 \%$.

Compound 5M: Yield: 40.6\%; ${ }^{1} \mathrm{H}-\mathrm{NMR}\left(300 \mathrm{MHz}, \mathrm{CDCl}_{3}, \delta\right.$, ppm): $0.96\left(\mathrm{t}, 3 \mathrm{H},-\mathrm{CH}_{2}-\mathrm{CH}_{3}\right), 1.31$ (s, $3 \mathrm{H},-\mathrm{CH}_{3}$ on the oxetane ring), $1.47-1.80\left(\mathrm{~m}, 12 \mathrm{H},-\mathrm{OCH}_{2}\left(\mathrm{CH}_{2}\right)_{2} \mathrm{CH}_{3} ;-\mathrm{OCH}_{2}\left(\mathrm{CH}_{2}\right)_{4} \mathrm{CH}_{2}-\right), 3.46$ $\left(\mathrm{t}, 4 \mathrm{H},-\mathrm{CH}_{2} \mathrm{OCH}_{2}-\right), 3.94\left(\mathrm{t}, 4 \mathrm{H},-\mathrm{CH}_{2}-\mathrm{OPh}-\right), 4.35,4.51$ (AB quartet, each $2 \mathrm{H},-\mathrm{CH}_{2}-$ on the oxetane ring), 6.86, $7.39\left(\mathrm{~m}, 6 \mathrm{H}, 4 \mathrm{H}\right.$, stilbene protons); element analysis: Calc. for $\mathrm{C}_{29} \mathrm{H}_{40} \mathrm{O}_{4}$ : C 76.99, $\mathrm{H}$ 8.85, O 14.16; found C 77.03, H 9.02, O 13.95\%.

Compound 6M: Yield: $36.7 \%$; ${ }^{1} \mathrm{H}-\mathrm{NMR}\left(300 \mathrm{MHz}, \mathrm{CDCl}_{3}, \delta, \mathrm{ppm}\right): 0.91\left(\mathrm{t}, 3 \mathrm{H},-\mathrm{CH}_{2}-\mathrm{CH}_{3}\right), 1.31$ (s, $3 \mathrm{H},-\mathrm{CH}_{3}$ on the oxetane ring), $1.41-1.79\left(\mathrm{~m}, 14 \mathrm{H},-\mathrm{OCH}_{2}\left(\mathrm{CH}_{2}\right)_{3} \mathrm{CH}_{3} ;-\mathrm{OCH}_{2}\left(\mathrm{CH}_{2}\right)_{4} \mathrm{CH}_{2}-\right), 3.45$ $\left(\mathrm{t}, 4 \mathrm{H},-\mathrm{CH}_{2} \mathrm{OCH}_{2}-\right), 3.94\left(\mathrm{t}, 4 \mathrm{H},-\mathrm{CH}_{2}-\mathrm{OPh}-\right), 4.35,4.50\left(\mathrm{AB}\right.$ quartet, each $2 \mathrm{H},-\mathrm{CH}_{2}-$ on the oxetane 
ring), 6.86, 7.39 (m, $6 \mathrm{H}, 4 \mathrm{H}$, stilbene protons); element analysis: Calc. for $\mathrm{C}_{30} \mathrm{H}_{42} \mathrm{O}_{4}$ : C 77.25, $\mathrm{H}$ 9.01, O 13.73; found C 77.21, H 9.15, O 13.64\%.

Compound 7M: Yield: 41.0\%; ${ }^{1} \mathrm{H}-\mathrm{NMR}\left(300 \mathrm{MHz}, \mathrm{CDCl}_{3}, \delta, \mathrm{ppm}\right): 0.91\left(\mathrm{t}, 3 \mathrm{H},-\mathrm{CH}_{2}-\mathrm{CH}_{3}\right)$, 1.31-1.80 (m, $19 \mathrm{H},-\mathrm{CH}_{3}$ on the oxetane ring, $\left.-\mathrm{OCH}_{2}\left(\mathrm{CH}_{2}\right)_{4} \mathrm{CH}_{3},-\mathrm{OCH}_{2}\left(\mathrm{CH}_{2}\right)_{4} \mathrm{CH}_{2}-\right), 3.46(\mathrm{t}, 4 \mathrm{H}$, $\left.-\mathrm{CH}_{2} \mathrm{OCH}_{2}-\right), 3.94\left(\mathrm{t}, 4 \mathrm{H},-\mathrm{CH}_{2}-\mathrm{OPh}-\right), 4.35,4.50$ (AB quartet, each $2 \mathrm{H},-\mathrm{CH}_{2}-$ on the oxetane ring), 6.86, $7.39\left(\mathrm{~m}, 6 \mathrm{H}, 4 \mathrm{H}\right.$, stilbene protons); element analysis: Calc. for $\mathrm{C}_{31} \mathrm{H}_{44} \mathrm{O}_{4}: \mathrm{C} 77.50, \mathrm{H}$ 9.17, O 13.33; found C 77.47, $\mathrm{H}$ 9.26, O $13.27 \%$.

Compound 8M: Yield: $40.6 \%$; ${ }^{1} \mathrm{H}-\mathrm{NMR}\left(300 \mathrm{MHz}, \mathrm{CDCl}_{3}, \delta, \mathrm{ppm}\right): 0.88\left(\mathrm{t}, 3 \mathrm{H},-\mathrm{CH}_{2}-\mathrm{CH}_{3}\right)$, 1.26-1.80 (m, $21 \mathrm{H},-\mathrm{CH}_{3}$ on the oxetane ring, $\left.-\mathrm{OCH}_{2}\left(\mathrm{CH}_{2}\right)_{5} \mathrm{CH}_{3},-\mathrm{OCH}_{2}\left(\mathrm{CH}_{2}\right)_{4} \mathrm{CH}_{2}-\right), 3.46(\mathrm{t}, 4 \mathrm{H}$, $\left.-\mathrm{CH}_{2} \mathrm{OCH}_{2}-\right), 3.95\left(\mathrm{t}, 4 \mathrm{H},-\mathrm{CH}_{2}-\mathrm{OPh}-\right), 4.35,4.50$ (AB quartet, each $2 \mathrm{H},-\mathrm{CH}_{2}-$ on the oxetane ring), 6.86, $7.39\left(\mathrm{~m}, 6 \mathrm{H}, 4 \mathrm{H}\right.$, stilbene protons); element analysis: Calc. for $\mathrm{C}_{32} \mathrm{H}_{46} \mathrm{O}_{4}$ : C 77.73, $\mathrm{H}$ 9.31, $\mathrm{O}$ 12.96; found C 77.60, H 9.40, O $13.00 \%$.

Compound 9M: Yield: $38.6 \% ;{ }^{1} \mathrm{H}-\mathrm{NMR}\left(300 \mathrm{MHz}, \mathrm{CDCl}_{3}, \delta, \mathrm{ppm}\right): 0.87\left(\mathrm{t}, 3 \mathrm{H},-\mathrm{CH}_{2}-\mathrm{CH}_{3}\right)$, 1.26-1.82 (m, $23 \mathrm{H},-\mathrm{CH}_{3}$ on the oxetane ring, $\left.-\mathrm{OCH}_{2}\left(\mathrm{CH}_{2}\right)_{6} \mathrm{CH}_{3},-\mathrm{OCH}_{2}\left(\mathrm{CH}_{2}\right)_{4} \mathrm{CH}_{2}-\right), 3.46(\mathrm{t}, 4 \mathrm{H}$, $\left.-\mathrm{CH}_{2} \mathrm{OCH}_{2}-\right), 3.94\left(\mathrm{t}, 4 \mathrm{H},-\mathrm{CH}_{2}-\mathrm{OPh}-\right), 4.35,4.50$ ( $\mathrm{AB}$ quartet, each $2 \mathrm{H},-\mathrm{CH}_{2}-$ on the oxetane ring), 6.86, $7.39\left(\mathrm{~m}, 6 \mathrm{H}, 4 \mathrm{H}\right.$, stilbene protons); element analysis: Calc. for $\mathrm{C}_{33} \mathrm{H}_{48} \mathrm{O}_{4}$ : C 77.95, $\mathrm{H}$ 9.45, $\mathrm{O}$ 12.60; found C 77.92, H 9.54, O $12.54 \%$.

Compound 10M: Yield: $34.7 \% ;{ }^{1} \mathrm{H}-\mathrm{NMR}\left(300 \mathrm{MHz}, \mathrm{CDCl}_{3}, \delta, \mathrm{ppm}\right): 0.96\left(\mathrm{t}, 3 \mathrm{H},-\mathrm{CH}_{2}-\mathrm{CH}_{3}\right)$, 1.29-1.79 $\left(\mathrm{m}, 27 \mathrm{H},-\mathrm{CH}_{3}\right.$ on the oxetane ring, $\left.-\mathrm{OCH}_{2}\left(\mathrm{CH}_{2}\right)_{2} \mathrm{CH}_{3},-\mathrm{OCH}_{2}\left(\mathrm{CH}_{2}\right)_{10} \mathrm{CH}_{2}-\right), 3.43(\mathrm{t}, 4 \mathrm{H}$, $\left.-\mathrm{CH}_{2} \mathrm{OCH}_{2}-\right), 3.96\left(\mathrm{t}, 4 \mathrm{H},-\mathrm{CH}_{2}-\mathrm{OPh}-\right), 4.35,4.50\left(\mathrm{AB}\right.$ quartet, each $2 \mathrm{H},-\mathrm{CH}_{2}-$ on the oxetane ring), 6.86, $7.39\left(\mathrm{~m}, 6 \mathrm{H}, 4 \mathrm{H}\right.$, stilbene protons); element analysis: Calc. for $\mathrm{C}_{35} \mathrm{H}_{52} \mathrm{O}_{4}$ : C 78.36, $\mathrm{H}$ 9.70, $\mathrm{O}$ 11.94; found C 78.22, H 9.80, O 11.98\%.

Compound 11M: Yield: 39.1\%; ${ }^{1} \mathrm{H}-\mathrm{NMR}\left(300 \mathrm{MHz}, \mathrm{CDCl}_{3}, \delta, \mathrm{ppm}\right): 0.91\left(\mathrm{t}, 3 \mathrm{H},-\mathrm{CH}_{2}-\mathrm{CH}_{3}\right)$, 1.27-1.78 (m, $29 \mathrm{H},-\mathrm{CH}_{3}$ on the oxetane ring, $\left.-\mathrm{OCH}_{2}\left(\mathrm{CH}_{2}\right)_{3} \mathrm{CH}_{3},-\mathrm{OCH}_{2}\left(\mathrm{CH}_{2}\right)_{10} \mathrm{CH}_{2}-\right), 3.43(\mathrm{t}, 4 \mathrm{H}$, $\left.-\mathrm{CH}_{2} \mathrm{OCH}_{2}-\right), 3.94\left(\mathrm{t}, 4 \mathrm{H},-\mathrm{CH}_{2}-\mathrm{OPh}-\right), 4.34,4.50$ ( $\mathrm{AB}$ quartet, each $2 \mathrm{H},-\mathrm{CH}_{2}-$ on the oxetane ring), 6.86, $7.39\left(\mathrm{~m}, 6 \mathrm{H}, 4 \mathrm{H}\right.$, stilbene protons); element analysis: Calc. for $\mathrm{C}_{36} \mathrm{H}_{54} \mathrm{O}_{4}$ : C 78.54, $\mathrm{H}$ 9.82, O 11.64; found C 78.26, H 9.90, O $11.84 \%$.

Compound 12M: Yield: $24.1 \% ;{ }^{1} \mathrm{H}-\mathrm{NMR}\left(300 \mathrm{MHz}, \mathrm{CDCl}_{3}, \delta, \mathrm{ppm}\right): 0.90\left(\mathrm{t}, 3 \mathrm{H},-\mathrm{CH}_{2}-\mathrm{CH}_{3}\right)$, 1.26-1.79 $\left(\mathrm{m}, 31 \mathrm{H},-\mathrm{CH}_{3}\right.$ on the oxetane ring, $\left.-\mathrm{OCH}_{2}\left(\mathrm{CH}_{2}\right)_{4} \mathrm{CH}_{3},-\mathrm{OCH}_{2}\left(\mathrm{CH}_{2}\right){ }_{10} \mathrm{CH}_{2}-\right), 3.43(\mathrm{t}, 4 \mathrm{H}$, $\left.-\mathrm{CH}_{2} \mathrm{OCH}_{2}-\right)$, $3.94\left(\mathrm{t}, 4 \mathrm{H},-\mathrm{CH}_{2}-\mathrm{OPh}-\right), 4.34,4.50$ (AB quartet, each $2 \mathrm{H},-\mathrm{CH}_{2}-$ on the oxetane ring), 6.86, $7.39\left(\mathrm{~m}, 6 \mathrm{H}, 4 \mathrm{H}\right.$, stilbene protons); element analysis: Calc. for $\mathrm{C}_{37} \mathrm{H}_{56} \mathrm{O}_{4}: \mathrm{C} 78.72, \mathrm{H} 9.93, \mathrm{O}$ 11.35; found C 78.40, H 10.05, O 11.55\%.

Compound 13M: Yield: $27.4 \% ;{ }^{1} \mathrm{H}-\mathrm{NMR}\left(300 \mathrm{MHz}, \mathrm{CDCl}_{3}, \delta, \mathrm{ppm}\right): 0.87\left(\mathrm{t}, 3 \mathrm{H},-\mathrm{CH}_{2}-\mathrm{CH}_{3}\right)$, 1.26-1.77 $\left(\mathrm{m}, 33 \mathrm{H},-\mathrm{CH}_{3}\right.$ on the oxetane ring, $\left.-\mathrm{OCH}_{2}\left(\mathrm{CH}_{2}\right)_{5} \mathrm{CH}_{3},-\mathrm{OCH}_{2}\left(\mathrm{CH}_{2}\right)_{10} \mathrm{CH}_{2}-\right), 3.44(\mathrm{t}, 4 \mathrm{H}$, $\left.-\mathrm{CH}_{2} \mathrm{OCH}_{2}-\right), 3.95\left(\mathrm{t}, 4 \mathrm{H},-\mathrm{CH}_{2}-\mathrm{OPh}-\right), 4.35,4.49\left(\mathrm{AB}\right.$ quartet, each $2 \mathrm{H},-\mathrm{CH}_{2}-$ on the oxetane ring), 6.85, 7.38 (m, $6 \mathrm{H}, 4 \mathrm{H}$, stilbene protons); element analysis: Calc. for $\mathrm{C}_{38} \mathrm{H}_{58} \mathrm{O}_{4}$ : C 78.90, $\mathrm{H}$ 10.03, O 11.07; found C 78.73, H 10.11, O 11.16\%.

Compound 14M: Yield: $34.7 \% ;{ }^{1} \mathrm{H}-\mathrm{NMR}\left(300 \mathrm{MHz}, \mathrm{CDCl}_{3}, \delta, \mathrm{ppm}\right): 0.89\left(\mathrm{t}, 3 \mathrm{H},-\mathrm{CH}_{2}-\mathrm{CH}_{3}\right)$, 1.29-1.81 (m, 35H, $-\mathrm{CH}_{3}$ on the oxetane ring, $\left.-\mathrm{OCH}_{2}\left(\mathrm{CH}_{2}\right)_{6} \mathrm{CH}_{3},-\mathrm{OCH}_{2}\left(\mathrm{CH}_{2}\right){ }_{10} \mathrm{CH}_{2}-\right), 3.46(\mathrm{t}, 4 \mathrm{H}$, $\left.-\mathrm{CH}_{2} \mathrm{OCH}_{2}-\right), 3.94\left(\mathrm{t}, 4 \mathrm{H},-\mathrm{CH}_{2}-\mathrm{OPh}-\right), 4.35,4.50\left(\mathrm{AB}\right.$ quartet, each $2 \mathrm{H},-\mathrm{CH}_{2}-$ on the oxetane ring), 6.86, $7.39\left(\mathrm{~m}, 6 \mathrm{H}, 4 \mathrm{H}\right.$, stilbene protons); element analysis: Calc. for $\mathrm{C}_{39} \mathrm{H}_{60} \mathrm{O}_{4}$ : C 79.05, $\mathrm{H} 10.14, \mathrm{O} 10.81$; found C 78.92, H 10.20, O 10.88\%.

\subsection{Synthesis of Polymers $\mathbf{1 P} \mathbf{1 4 P}$}

In this study, all the polymers were synthesized by cationic ring-opening polymerization. The preparation of polymer is described below. Under nitrogen, dichloromethane was dried by calcium hydride and was distilled just prior to use. Boron trifluoride ether complex (freshly distilled) was used as an initiator. Under nitrogen, a solution of monomer $(0.5 \mathrm{mmol})$ and dichloromethane $(5 \mathrm{~mL})$ was cooled to $0{ }^{\circ} \mathrm{C}$ and the initiator of $2 \%$ mol with respect to monomer was injected with a syringe. The reaction solution mixture was stirred at $0{ }^{\circ} \mathrm{C}$ for $24 \mathrm{~h}$. Then, the resulting polymers were 
precipitated in methanol and purified further by dissolving in dichloromethane and then precipitating in ethanol repeatedly. The absence of monomer was checked by ${ }^{1} \mathrm{H}-\mathrm{NMR}$ and GPC.

\section{Results and Discussion}

This study intends to explore the liquid crystalline monomers of oxetane and liquid crystalline polyoxetane. Among the compounds of these monomers and polymers, spacer length and terminal chain length have influences on the thermal properties and mesophase. The molecular structure and the general synthetic procedures of the series of monomers are shown in Scheme 1, and all the products are examined by the nuclear magnetic resonance spectrometer and elemental analyzer in order to verify the correction of the molecular structure. There is one most important part among all the monomers in terms of using nuclear magnetic resonance spectrometer, that is oxetane in which the hydrogen, located on two carbons being beside the oxygen atoms, is the split from $\mathrm{AB}$ quartet, and it will gradually be disappearing after the ring-opening polymerization.

Monomers are composed of trans-stilbene as a mesogenic unit, their spacers length include several different alkyl chains $(\mathrm{n}=3,4,5,6,12)$ and links with the terminals of different alkoxyl length $(\mathrm{m}=1$, $4,5,6,7,8)$. Moreover, there are fourteen monomers $(\mathbf{1} \mathbf{M} \sim \mathbf{1 4} \mathbf{M})$ in this series, and their structures are exhibited in Scheme 1.

All monomers (1M 14M) used BF3. OEt2 as an initiator to carry out the ring-opening polymerization and successfully synthesized a series of brand-new side-chain liquid crystalline polymers (1P $\sim \mathbf{1 4 P})$, and their structures and their synthesis procedures are listed in Scheme 1.

The thermal and mesomorphic properties of monomers $(\mathbf{1 M} \sim \mathbf{1 4 M})$ and polymers $(\mathbf{1 P} \sim \mathbf{1 4 P})$ are measured using DSC, POM, and X-ray. The phase transition temperature and enthalpy changed of monomers $\mathbf{1 M} \sim \mathbf{1 4} \mathbf{M}$ are reported in Table 1 . The series of monomers $(\mathbf{1} \mathbf{M} \sim \mathbf{1 4} \mathbf{M})$ reveal enantiotropic smectic $\mathrm{H}$ and smectic $\mathrm{G}$ phases.

There is no obvious regularity in the change of the phase transition temperature of the monomer $\mathbf{1 M} \sim \mathbf{M}$, although the length of the spacer changes. This phenomenon may be affected by the volume of oxetane.

Table 1 illustrates the representative phase transition temperature of monomers $\mathbf{5 M} \sim \mathbf{9 M}$ (containing six methylene units spacer length). As can be seen from Table 1 the tendency toward mesomorphic temperature range increase by increasing the length of alkoxy terminal group ( $5 \mathbf{M}$ is $2.6{ }^{\circ} \mathrm{C}, \mathbf{6 M}$ is $3.3^{\circ} \mathrm{C}, 7 \mathbf{M}$ is $2.1^{\circ} \mathrm{C}, 8 \mathbf{M}$ is $20.6{ }^{\circ} \mathrm{C}, 9 \mathbf{M}$ is $38.1{ }^{\circ} \mathrm{C}$ ). In addition, when the length of the terminal groups become shorter, the transition temperature of liquid crystalline will gradually overlap with the isotropic temperature. $5 \mathbf{M} \sim 7 \mathbf{M}$ are observed by POM, only the tiny transition of liquid crystalline phases can be seen. There is a tendency for the isotropic temperature to lower down the temperature from 136.4 to $118.0^{\circ} \mathrm{C}$ when the length of terminal alkoxy is changed from $\mathrm{m}=4$ to $\mathrm{m}$ $=8.10 \mathrm{M} \sim 14 \mathrm{M}$ (containing twelve methylene units spacer length) finds that the length of terminal alkoxy becomes larger, and the mesogenic temperature range becomes smaller $\left(\mathbf{1 0 M}\right.$ is $77.9^{\circ} \mathrm{C}, \mathbf{1 1} \mathbf{M}$ is $56.9^{\circ} \mathrm{C}, \mathbf{1 2} \mathrm{M}$ is $32.2{ }^{\circ} \mathrm{C}, \mathbf{1 3} \mathrm{M}$ is $22.6^{\circ} \mathrm{C}, \mathbf{1 4 M}$ is $16.0^{\circ} \mathrm{C}$ ). This is the opposite result as compared with that of monomers $5 \mathbf{M} \sim 9 \mathbf{M}$. This result may be due to the flexible spacer with a too larger length. From the above result, it is found that the mesomorphic temperature range has a great relationship with the spacer length.

Taking $9 \mathrm{M}$ for an example, the dendritic growth pattern is developed at the temperature of $116.1^{\circ} \mathrm{C}$ during the cooling scanning process, then mosaic platelets (Figure 1A) is formed at the temperature of $115.6{ }^{\circ} \mathrm{C}$. At last, the liquid crystalline mesophase includes a Zig-Zag line shown at the diagram of mosaic platelets (Figure 1B) at the temperature of $82.6{ }^{\circ} \mathrm{C}$. This characteristic is a transition phenomenon from smectic $\mathrm{G}$ phases to smectic $\mathrm{H}$ phases. In addition, in the X-ray test, Figure 2 presents the $X$-ray diffraction diagrams obtained from the powder sample of $9 \mathrm{M}$ at $54.6^{\circ} \mathrm{C}$. The diffraction diagram presents a sharp first-order reflection at $34.53 \AA$, which is a layer of the length of smectic phase, and there are three diffraction peaks of $4.513,4.240$, and $3.995 \AA$ in terms of the wider angles. Most of the X-ray diffraction studies performed on the $\mathrm{SH}$ phase indicate that it has 
a structure equivalent to that of the SE phase, except that the molecules have their long axes tilted concerning the normal to the layer planes. From the description of the phase as being of the SE type, it must be assumed that the molecules adopt an orthorhombic close-packing in a plane at right angles to the molecular long axes. Hence, because of the tilt, the pseudo-hexagonal net becomes even more distorted, and the phase has a monoclinic structure. According to the research of Volino, Dianoux and Hervet [17], the results of the $\mathrm{X}$-ray test and the diagram represent smectic $\mathrm{H}$ phase.

Table 1. Phase transition temperature and thermodynamic parameters of monomers $\mathbf{1 M} \sim \mathbf{1 4}$.

\begin{tabular}{|c|c|}
\hline Compound & $\begin{array}{c}\text { Phase Transitions, }{ }^{\circ} \mathrm{C} \text { (Corresponding Enthalpy Changes, } \\
\text { Kcal } / \text { mol) } \\
\frac{\text { heating }}{\text { cooling }}\end{array}$ \\
\hline $1 \mathrm{M}$ & $\frac{S_{H} 108.5(-)^{\mathrm{a}} S_{G} 117.1(4.63) I}{I 111.6(-4.73) S_{G} 102.0(-)^{\mathrm{a}} S_{H}}$ \\
\hline $2 \mathrm{M}$ & $\frac{S_{H} 121.3(-)^{\mathrm{a}} S_{G} 124.5(3.92) I}{I 120.9(-3.82) S_{G} 116.0(-)^{\mathrm{a}} S_{H}}$ \\
\hline $3 \mathbf{M}$ & $\frac{S_{H} 90.0(-)^{\mathrm{a}} S_{G} 118.0(3.68) I}{I 113.0(-3.90) S_{G} 81.6(-)^{a} S_{H}}$ \\
\hline $4 \mathrm{M}$ & $\frac{S_{H} 109.3(-)^{\mathrm{a}} S_{G} 114.3(3.97) I}{I 104.9(-4.40) S_{G} 101.6(-)^{\mathrm{a}} S_{H}}$ \\
\hline $5 \mathrm{M}$ & $\frac{S_{H} 132.1(-)^{\mathrm{a}} S_{G} 136.4(6.94) I}{I 132.6(-7.37) S_{G} 130.0(-)^{\mathrm{a}} S_{H}}$ \\
\hline $6 \mathrm{M}$ & $\frac{S_{H} 130.8(-)^{\mathrm{a}} S_{G} 132.2(8.05) I}{I 130.6(-8.20) S_{G} 127.3(-)^{\mathrm{a}} S_{H}}$ \\
\hline $7 \mathbf{M}$ & $\frac{S_{H} 124.8(-)^{\mathrm{a}} S_{G} 126.2(4.77) I}{I 123.9(-5.10) S_{G} 121.8(-)^{\mathrm{a}} S_{H}}$ \\
\hline $8 \mathrm{M}$ & $\frac{S_{H} 121.9(-)^{\mathrm{a}} S_{G} 122.8(9.52) I}{I 117.6(-7.77) S_{G} 97.7(-0.72) S_{H}}$ \\
\hline $9 \mathrm{M}$ & $\frac{S_{H} 97.0(0.72) S_{G} 118.0(5.81) I}{I 114.2(-6.37) S_{G} 76.1(-0.29) S_{H}}$ \\
\hline $10 \mathrm{M}$ & $\frac{S_{H} 66.1(0.73) S_{G} 131.6(7.19) I}{I 126.7(-7.45) S_{G} 48.8(-0.77) S_{H}}$ \\
\hline $11 M$ & $\frac{S_{H} 64.0(1.29) S_{G} 119.8(5.06) I}{I 114.9(-5.14) S_{G} 58.0(-1.02) S_{H}}$ \\
\hline $12 \mathrm{M}$ & $\frac{S_{H} 81.7(-)^{\mathrm{a}} S_{G} 116.6(8.08) I}{I 110.0(-7.59) S_{G} 77.8(-)^{\mathrm{a}} S_{H}}$ \\
\hline $13 \mathrm{M}$ & $\frac{S_{H} 97.1(-)^{\mathrm{a}} S_{G} 121.2(8.86) I}{I 115.8(-9.84) S_{G} 93.2(-)^{\mathrm{a}} S_{H}}$ \\
\hline $14 \mathrm{M}$ & $\frac{S_{H} 106.4(-)^{\mathrm{a}} S_{G} 123.5(14.0) I}{I 119.1(-13.6) S_{G} 103.1(-)^{\mathrm{a}} S_{H}}$ \\
\hline
\end{tabular}

$S_{A}=$ smectic A, $S_{E}=$ smectic E, $I=$ isotropic. ${ }^{\text {a }}$ determined by optical polarizing microscopic observation.

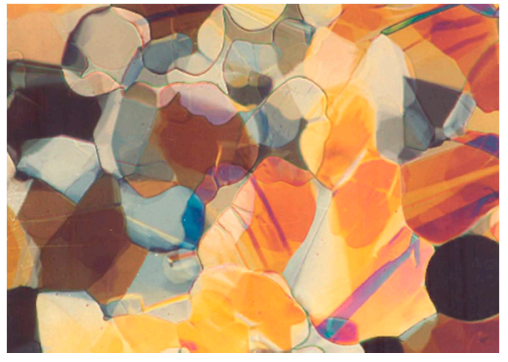

(A)

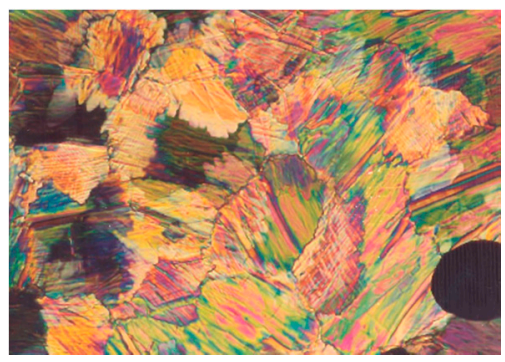

(B)

Figure 1. Optical polarizing micrographs displayed by monomer $9 \mathrm{M}$ upon cooling from isotropic phase. (A) The smectic $\mathrm{G}$ mosaic platelets texture obtained at $115.6{ }^{\circ} \mathrm{C}(320 \times)$. (B) The smectic $\mathrm{H}$ zig-zag line texture obtained at $82.6{ }^{\circ} \mathrm{C}(320 \times)$. 


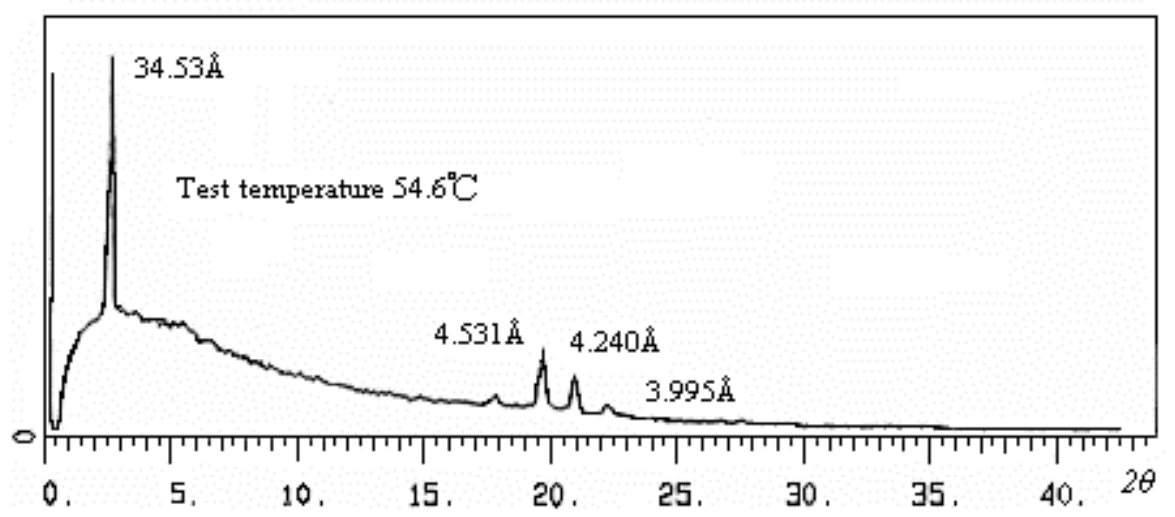

Figure 2. X-ray diffraction trace of $9 \mathbf{M}$.

Table 2 reports the thermal transition and thermodynamic parameters of polymers $\mathbf{1 P} \sim \mathbf{1 4 P}$. Polymers 1P 4P and 6P 11P exhibit enantiotropic smectic A phase, 5P and 12P 14P show enantiotropic smectic A and smectic E phase. Figure 3A,B exhibits the texture of polymer 14P by optical polarizing micrograph. Figure $3 \mathrm{~A}$ shows a focal conic fan texture of smectic A phase at $172.6^{\circ} \mathrm{C}$. Figure $3 \mathrm{~B}$ displays the fissure focal cone fan shape of the texture of smectic $\mathrm{E}$ phase at $152.2^{\circ} \mathrm{C}$, while the temperature is cooling to $120^{\circ} \mathrm{C}$, the texture does not change except in the bigger crack. Therefore, it can be a regular liquid crystalline smectic texture of crystallization. Seen from Table 2, polymers (1P $\sim \mathbf{4 P}$ ) that contain a short flexible spacer $(n=3 \sim 6)$ and only one methyl terminal group show smectic A phase. Polymers (5P 9P, six methylene units spacer length; 10P 14P, twelve methylene units spacer length) have different length of alkoxy terminal groups $(\mathrm{m}=4 \sim 8)$. Their isotropic temperature and liquid crystalline transition temperature exhibits the same tendency. The isotropic temperature of $5 \mathbf{P}$ and 10P $(\mathrm{m}=4)$ is especially high, maybe four alkoxy terminal groups can be easily arranged. 6P $\sim 9 \mathbf{P}$ and 11P 14P $(\mathrm{m}=5 \sim 8)$ polymers increase in isotropic temperature with the length of the alkoxy terminal group. 11P 14P more than $6 \mathrm{P} \sim 10 \mathrm{P}$ formed the regular liquid crystalline phase because they have a long flexible spacer.

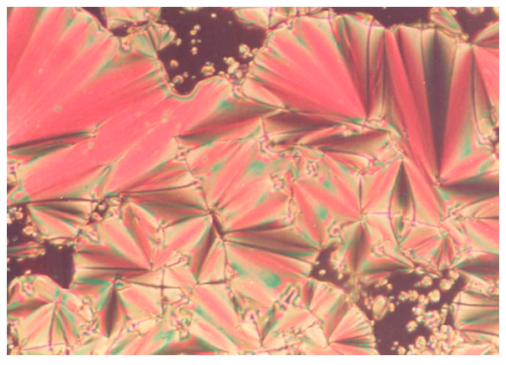

(A)

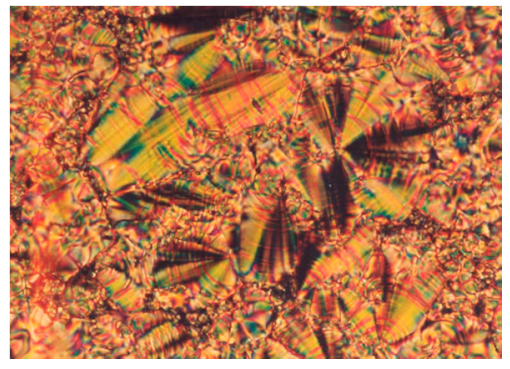

(B)

Figure 3. Optical polarizing micrographs displayed by polymer 14P upon cooling from the isotropic phase. (A) The smectic A focal-conic texture obtained at $172.6^{\circ} \mathrm{C}(320 \times)$. (B) The smectic E banded focal-conic texture obtained at $152.2^{\circ} \mathrm{C}(320 \times)$.

The synthesized polymers (1P $\sim \mathbf{1 4 P}$ ) cannot be solved in an organic solvent (such as hexane, toluene, m-cresol, methanol, dichloromethane, acetone, ethyl acetate, THF, DMF, DMSO) under room temperature. During the ring-opening polymerization, the stilbene group might be cross-linked, because the total amount of $\mathrm{H}$ was decreased from the NMR spectrum after the polymerization, which might also cause the poor solubility of the synthesized polymer. In this study, $7 \mathbf{P}$ and $\mathbf{1 4 P}$ were selected for measuring molecular weight under the higher temperature of GPC, in which the solvent of $\mathrm{m}$-cresol was used, and the test temperature of $85^{\circ} \mathrm{C}$ was set. The molecular weight of $7 \mathrm{P}$ is $M_{\mathrm{n}}=4031, M_{\mathrm{w}}=5107, \mathrm{DPI}=1.266$. The molecular weight of $14 \mathrm{P}$ is $M_{\mathrm{n}}=3666, M_{\mathrm{w}}=5052$, 
$\mathrm{DPI}=1.378$. The results are presented as a ring-polymerization reaction by BF3. OEt2, the molecular weight of the obtained polymer is not high, but its dpi is small, as expected. The structure of the polymers is comb-like, and polystyrene was used as the standard of GPC, the test results from using GPC would be lower. However, in fact, the molecule weight should be higher.

Table 2. Phase transition temperature and thermodynamic parameters of polymers $\mathbf{1 P} \sim \mathbf{1 8 P}$.

\begin{tabular}{|c|c|}
\hline Compound & $\begin{array}{c}\text { Phase Transitions, }{ }^{\circ} \mathrm{C} \text { (Corresponding Enthalpy Changes, } \\
\text { Kcal/mol) } \\
\frac{\text { heating }}{\text { cooling }}\end{array}$ \\
\hline $1 P$ & $\frac{K 174.1(-)^{\mathrm{a}} S_{A} 178.0(4.08) I}{I 173.6(-3.82) S_{A} 170.2(-)^{\mathrm{a}} K}$ \\
\hline $2 P$ & $\frac{K 185.1(-)^{\mathrm{a}} S_{A} 189.7(4.98) I}{I 186.6(-4.90) S_{A} 178.6(-)^{\mathrm{a}} K}$ \\
\hline $3 P$ & $\frac{K 156.0(-)^{\mathrm{a}} S_{A} 152.2(4.52) I}{I 146.6(-4.56) S_{A} 135.4(-)^{\mathrm{a}} K}$ \\
\hline $4 P$ & $\frac{K 121.5(-)^{\mathrm{a}} S_{A} 126.7(2.93) I}{I 125.7(-2.61) S_{A} 115.8(-)^{\mathrm{a}} K}$ \\
\hline $5 P$ & $\frac{K 171.0(-)^{\mathrm{a}} S_{E} 188.5(-)^{\mathrm{a}} S_{A} \text { 193.1(5.26) I }}{I \text { 191.6(-4.83) } S_{A} 187.4(-)^{\mathrm{a}} S_{E} 168.5(-)^{\mathrm{a}} K}$ \\
\hline $6 P$ & $\frac{K 166.4(-)^{\mathrm{a}} S_{A} 176.8(7.44) I}{I 172.6(-6.99) S_{A} 167.0(-)^{\mathrm{a}} K}$ \\
\hline $7 P$ & $\frac{K 168.4(-)^{\mathrm{a}} S_{A} 179.5(8.53) I}{I 174.6(-7.66) S_{A} 170.1(-)^{\mathrm{a}} K}$ \\
\hline $8 P$ & $\frac{K 168.7(-)^{\mathrm{a}} S_{A} 179.7(7.80) I}{I 173.8(-7.60) S_{A} 166.3(-)^{\mathrm{a}} K}$ \\
\hline 9P & $\frac{K 174.0(-)^{\mathrm{a}} S_{A} 183.9(7.27) I}{I 176.4(-6.80) S_{A} 167.3(-)^{\mathrm{a}} \mathrm{K}}$ \\
\hline $10 P$ & $\frac{K 169.8(-)^{\mathrm{a}} S_{A} 183.8(6.76) I}{I 177.3(-7.11) S_{A} 165.4(-)^{\mathrm{a}} K}$ \\
\hline 11P & $\frac{K_{1} 69.7(0.38) K_{2} 90.4(0.27) K_{3} 133.7(-)^{\mathrm{a}} S_{A} 138.6(6.78) I}{I 130.4(-7.29) S_{A} 122.4(-)^{\mathrm{a}} K_{2} 61.5(-0.55) K_{1}}$ \\
\hline $12 P$ & $\frac{K_{1} 84.6(0.46) K_{2} 126.7(1.81) S_{E} 162.1(-)^{\mathrm{a}} S_{A} 167.9(0.19) I}{I 164.4(-0.53) S_{A} 159.6(-)^{\mathrm{a}} S_{E} 123.1(-0.29) K_{2} 61.6(-0.53) K_{1}}$ \\
\hline $13 P$ & $\frac{K_{1} 93.9(1.42) K_{2} 161.4(-)^{\mathrm{a}} S_{E} 167.3(-)^{\mathrm{a}} S_{A} 175.3(6.88) I}{I 168.8(-7.19) S_{A} 163.1(-)^{\mathrm{a}} S_{E} 158.5(-)^{\mathrm{a}} K_{2} 89.3(-0.88) K_{1}}$ \\
\hline 14P & $\frac{K_{1} 101.0(1.12) K_{2} 164.5(-)^{\mathrm{a}} S_{E} 169.4(-)^{\mathrm{a}} S_{A} 177.4(7.08) I}{I 171.6(-7.18) S_{A} 164.8(-)^{\mathrm{a}} S_{E} 161.6(-)^{\mathrm{a}} K_{2} 98.2(-0.67) K_{1}}$ \\
\hline
\end{tabular}

\section{Conclusions}

In this study, a series of fourteen liquid crystalline monomers and polyoxetanes containing trans-biphenyl side group are successfully synthesized. It is known from the results that the liquid crystal phase transition temperature, the isotropic temperature, the stability of the liquid crystal phase, and the type of the liquid crystal all have a great influence on both the length of the soft spacer and the length of the terminal alkyl group. It is found that the difference in the length of the soft spacer and the length of the terminal alkyl group affect the monomer or polymer. When the length of the soft spacer and the length of the terminal alkyl group are increased, the liquid crystal phase formed is polymorphism of mesophases, such as $14 \mathrm{M}$ shows $S_{H}$ and $S_{G}, 14 \mathrm{P}$ shows $S_{A}$ and $S_{E}$, and has a relatively stable liquid crystal phase.

Author Contributions: Conceptualization, C.-H.L. and L.-C.W.; methodology, C.-C.C. and L.-C.W.; validation, C.-H.L. and L.-C.W.; formal analysis, C.-H.L.; data curation, C.-H.L.; writing-original draft preparation, C.-H.L. and L.-C.W.; writing-review and editing, C.-H.L.; funding acquisition, C.-C.C. and C.-H.L. All authors have read and agreed to the published version of the manuscript.

Funding: This research was funded by Chang Gung University of Science and Technology EZRPF3I0051. 
Acknowledgments: The authors are grateful for Chang Gung University of Science and Technology.

Conflicts of Interest: The authors declare no conflict of interest.

\section{References}

1. Finkelmann, H.; Rehage, G. Investigations on liquid crystalline polysiloxanes, Optical properties of cholesteric phases and influence of the flexible spacer on the mobility of the mesogenic groups. Makromol. Chem. Rapid Commun. 1980, 1, 733-740. [CrossRef]

2. Finkelmann, H.; Rehage, G. Investigations on liquid crystalline polysiloxanes, Cholesteric homopolymers-Synthesis and optical characterization. Makromol. Chem. Rapid Commun. 1982, 3, 859-864. [CrossRef]

3. Janini, G.M.; Laub, R.J.; Shaw, T.J. Synthesis and properties of high temperature mesomorphic polysiloxane (MEPSIL) solvents. Amide, ester and Schiff's base linked systems. Makromol. Chem. Rapid Commun. 1985, 6, 57-63. [CrossRef]

4. Bradshaw, J.S.; Schregenberger, C.; Chang, K.H.C.; Markides, K.E.; Lee, M.L. Synthesis and chromatographic properties of polysiloxane stationary phases containing biphenylcarboxylate ester liquid-crystalline side groups. J. Chromatogr. A 1986, 358, 95-106. [CrossRef]

5. Lin, C.H. Cholesteric Liquid Crystalline Copolymers for Gas Chromatographic Separation of Polycyclic Aromatic Compounds. Adv. Mater. Sci. Eng. 2012, 2012. [CrossRef]

6. Kaempf, G. Special Polymers for Data Memories. Polym. J. 1987, 19, 257-268. [CrossRef]

7. Coles, H.J.; Simon, R. Side chain polysiloxane liquid crystals and their behaviour in electric fields. Polymer 1986, 27, 811-816.

8. Wang, R.; Wang, Z.G. Theory of Side-Chain Liquid Crystal Polymers: Bulk Behavior and Chain Conformation. Macromolecules 2010, 43, 10096-10106. [CrossRef]

9. Zhang, B.Y.; Meng, F.B.; He, X.Z.; Lin, D.A. Synthesis and characterization of side chain liquid crystalline polymers exhibiting cholesteric and blue phases. Liq. Cryst. 2005, 32, 1161-1167. [CrossRef]

10. Reddy, G.S.M.; Jayaramudu, J.; Ray, S.S.; Varaprasad, K.; Sadiku, E.R. Side Chain Liquid Crystalline Polymers: Advances and Applications. Liq. Cryst. Polym. 2015, 2, 389-415.

11. Kawakami, Y.; Takahashi, K. Smectic liquid crystalline polyoxetane with novel mesogenic group. Polym. Bull. 1991, 25, 439-442. [CrossRef]

12. Kawakami, Y.; Takahashi, K.; Hibino, H. Synthesis of liquid crystalline polymers with a polyoxetane main chain. Macromolecules 1991, 24, 4531-4537. [CrossRef]

13. Kawakami, Y.; Nishiguchi, K.T.S.; Toida, K. Synthesis and thermal transition of side-chain liquid crystalline polyoxetanes having laterally attached mesogenic group. Polym. Int. 1993, 31, 35-40. [CrossRef]

14. Kawakami, Y.; Kishimoto, N. Synthesis of new disiloxane-containing polymers. Proc. Jpn. Acad. 1998, 74, 41-45. [CrossRef]

15. Campo, A.D.; Bello, A.; Perez, E.; Benavente, R. Liquid crystalline polyoxetanes with two mesogens in the side chain separated by a flexible spacer. Ferroelectrics 2000, 243, 137-144. [CrossRef]

16. Lee, J.W.; Oh, D.K.; Yelamaggad, C.V.; Nagamani, S.A.; Jin, J.I. Ferroelectric liquid crystalline polyoxetanes bearing chiral dimesogenic pendants. J. Mater. Chem. 2002, 12, 2225-2230. [CrossRef]

17. Volino, F.; Dianoux, A.J.; Hervet, H.J. Neutron Quasi-Elastic Scattering Study of Rotational Motions in the Smectic C, H and VI Phases of Terephtal-Bis-Butyl-Antline (TBBA). J. Phys. 1976, 37, 55-64. [CrossRef] 